\title{
Measuring hadron properties at finite temperature
}

\author{
David Seibert* and Charles Gale ${ }^{\dagger}$ \\ Physics Department, McGill University, Montréal, QC, H3A 2T8, Canada
}

(March 1995)

\begin{abstract}
We estimate the numbers and mass spectra of observed lepton and kaon pairs produced from $\phi$ meson decays in the central rapidity region of an $\mathrm{Au}+\mathrm{Au}$ collision at lab energy $11.6 \mathrm{GeV} /$ nucleon. The following effects are considered: possible mass shifts, thermal broadening due to collisions with hadronic resonances, and superheating of the resonance gas. Changes in the dilepton mass spectrum may be seen, but changes in the dikaon spectrum are too small to be detectable.
\end{abstract}

*Electronic mail (internet): seibert@hep.physics.mcgill.ca.

$\dagger$ Electronic mail (internet): gale@hep.physics.mcgill.ca. 


\section{INTRODUCTION}

There is currently a great deal of interest in finite-temperature properties of hadronic resonances [1 7]. Recently, preliminary results for the $\phi$ meson mass spectrum in $\mathrm{Si}+\mathrm{Au}$ collisions at lab energy $11.6 \mathrm{GeV} /$ nucleon were obtained at Brookhaven's Alternating Gradient Synchrotron (AGS), by reconstructing the $\phi$ mesons from final-state kaon pairs [8]. No change was observed from the vacuum width of the $\phi$, although a possible small mass shift was observed for the most central events. These negative preliminary results could dampen the enthusiasm of other groups to study the $\phi$ peak in the dilepton channel. We show here that no observable change is expected for the $\phi$ peak in the dikaon spectrum, but that effects may be visible in the dilepton spectrum. Thus, there is still good reason to study the dilepton $\phi$ peak at the AGS, in spite of the fact that dikaon results are negative.

\section{EQUATION OF STATE}

The behavior of the hot matter is somewhat more complicated in events at AGS energies than in ultra-relativistic events, due to the large baryon densities and the lack of strangeness equilibration. The equation of state used here is almost the same as those used in Refs. [9 11. We describe the system using the temperature $T$, the baryon chemical potential $\mu_{B}$, and the strangeness and antistrangeness chemical potentials, respectively $\mu_{S}$ and $\mu_{\bar{S}}$, using the high energy conventions $c=\hbar=k_{B}=1$.

The matter has nearly isospin zero, so the up and down quark chemical potentials, respectively $\mu_{u}$ and $\mu_{d}$, are $\mu_{u}=\mu_{d}=\mu_{B} / 3$. The $u$ and $d$ quarks are approximately in chemical equilibrium with their antiquarks, so the $\bar{u}$ and $\bar{d}$ chemical potentials are $\mu_{\bar{u}}=$

$\mu_{\bar{d}}=-\mu_{B} / 3$. Finally, the net strangeness is also zero, since strong interactions conserve strangeness and the time scales are too short to allow weak interactions to be significant. The $s$ and $\bar{s}$ chemical potentials are respectively $\mu_{s}=\mu_{S}+\mu_{B} / 3$ and $\mu_{\bar{s}}=\mu_{\bar{S}}-\mu_{B} / 3$, so that $\mu_{\bar{S}}=\mu_{S}+2 \mu_{B} / 3$ [12]. It is unlikely that $s$ and $\bar{s}$ are in chemical equilibrium with each other, because $V R_{s} \ll n_{s} d V / d t$, where $V$ is the volume of the hot matter, $R_{s}$ is the production rate of $s$ quarks per unit volume, $n_{s}$ is the density of $s$ quarks, and $t$ is time. However, we assume for simplicity that the system is in chemical equilibrium, so that $\mu_{S}=-\mu_{B} / 3$; this assumption will be relaxed in later works. The mean number of $s$ quarks per event is much greater than unity, so the possible $s \bar{s}$ pair chemical potential |12 is omitted.

We model the QGP as a collection of free quarks and gluons, with a constant (bag) energy density $B \simeq(220 \mathrm{MeV})^{4}$. The low-temperature phase is treated as a resonance gas $(\mathrm{RG})$, using all confirmed strongly-interacting particles with known quantum numbers and without $c$ or $b$ content [13]. The chemical potential for resonance $i$ is

$$
\mu_{i}=\left(\lambda_{i}^{(u)}+\lambda_{i}^{(d)}+\lambda_{i}^{(s)}-\lambda_{i}^{(\bar{u})}-\lambda_{i}^{(\bar{d})}-\lambda_{i}^{(\bar{s})}\right) \mu_{B} / 3+\lambda_{i}^{(s)} \mu_{S}+\lambda_{i}^{(\bar{s})} \mu_{\bar{S}},
$$

where $\lambda_{i}^{(q)}$ is the number of constituent quarks of species $q$ in resonance $i$, and the chemical potentials correspond to those in the QGP. We take an excluded volume, $v_{\text {exc }}=4 \pi R_{\text {exc }}^{3} / 3$, for every resonance in the RG phase [9]. Our justification for this is that when the resonances 
overlap, the region where this occurs should be in the QGP phase, so we do not allow overlap in the RG phase.

The transition temperature, $T_{c}$, is obtained by setting the pressures equal in the two phases, with all chemical potentials fixed. In Fig. 1, we show the transition temperature as a function of $\mu_{B}$, for the cases (i) $n_{s}=n_{\bar{s}}, \mu_{\bar{S}}=-\mu_{S}$ (strangeness equilibrium under the strong interactions), and (ii) $\mu_{\bar{S}}=\mu_{S} \rightarrow-\infty$ (complete strangeness suppression). We give results for $R_{e x c}=0.5$ and $1 \mathrm{fm}$ in each case, adjusting the bag constant to give $T_{c}=150 \mathrm{MeV}$ for $\mu_{B}=0$; for the equilibrium case, we take $B^{1 / 4}=217$ and $220 \mathrm{MeV}$ for $R_{e x c}=0.5$ and 1 $\mathrm{fm}$ respectively, while for the case of complete strangeness suppression we take $B^{1 / 4}=207$ and $210 \mathrm{MeV}$. Here (and for the remainder of this paper) we evaluate all momentum integrals numerically to one percent accuracy.

We fix $\mu_{S}$ for the strangeness equilibrium curves by requiring that the QGP or RG be strangeness-neutral $\left(n_{\bar{s}}=n_{s}\right)$. At fixed $\mu_{B}$, the transition from one strangeness-neutral phase to the other phase (of arbitrary strangeness) occurs at almost exactly the same temperature whether the initial phase is QGP or RG, so we only show RG curves. This result, first noted in Refs. [9.10], is somewhat surprising, as the value of $\mu_{S}$ in the RG depends on the strange hadron spectrum, so that the transition temperatures could easily be very different for the two phases.

\section{EVOLUTION OF THE HOT MATTER}

We model the initial evolution following Ref. [14]. We approximate the nucleon wavefunctions to be constant inside cylinders with radius $r_{N}$ and length (parallel to the beam direction) $l_{N}$, and zero outside these cylinders. The radius is given by the nuclear radius, $r_{N}=7 \mathrm{fm}$ for $\mathrm{Au}$, while the length in the center of momentum $(\mathrm{cm})$ frame is $l_{N} \approx r_{N} / \gamma$. Here $\gamma=\left(1-v^{2}\right)^{-1 / 2}$, where $v \approx 1$ is the nuclear velocity in the $\mathrm{cm}$ frame; for fixed target collisions at beam energy $11.6 \mathrm{GeV} /$ nucleon, $\gamma=2.7$. In principle, $l_{N}$ is bounded from below by the minimum parton wavelength, of order $m_{\pi}^{-1}=1.4 \mathrm{fm}$, but for the collisions considered here $l_{N}=2.6 \mathrm{fm}$ so this lower bound is unimportant.

We then assume that the nucleon properties are unchanged during the collision, and neglect collisions of secondary particles. The rate of production of any quantity during the collision is then proportional to the overlap of the nucleon wavefunctions. For example, if the cylinders first touch at proper time $\tau=\sqrt{t^{2}-z^{2}}=0$, where $z$ is the position along the beam axis, the baryon rapidity density at rapidity $y=0$ is

$$
d N_{B} / d y=k_{B} l_{N}^{2} \times \begin{cases}0, & \tau_{*}<0, \\ \tau_{*}^{2}, & 0<\tau_{*}<1 / 2, \\ {\left[1-2\left(1-\tau_{*}\right)^{2}\right] / 2,} & 1 / 2<\tau_{*}<1, \\ 1 / 2, & 1<\tau_{*},\end{cases}
$$

where $k_{B}$ is an unspecified normalization constant, and $\tau_{*}=\tau / l_{N}$. The volume of hot matter per unit rapidity is approximately $\pi r_{N}^{2} \tau$, so the baryon density at $y=0$ is 


$$
n_{B}=\frac{d N_{B} / d y}{\pi r_{N}^{2} l_{N}} \times \begin{cases}0, & \tau_{*}<0 \\ 2 \tau_{*}, & 0<\tau_{*}<1 / 2, \\ {\left[1-2\left(1-\tau_{*}\right)^{2}\right] / \tau_{*},} & 1 / 2<\tau_{*}<1 \\ 1 / \tau_{*}, & 1<\tau_{*},\end{cases}
$$

where $d N_{B} / d y$ is the observed baryon rapidity density at $y=0$.

For $\tau>l_{N}$, we evolve the hot matter hydrodynamically, maintaining boost-invariance (and hence neglecting transverse expansion). We assume strong superheating in the RG phase, so that the hot matter never makes a transition to QGP, as the temperature never rises very far above $T_{c}$. The hot matter then follows the boost-invariant hydrodynamic equations [15]

$$
\begin{aligned}
\frac{d e}{d \tau} & =\frac{-(e+P)}{\tau}, \\
\frac{d n_{B}}{d \tau} & =\frac{-n_{B}}{\tau}, \\
\frac{d\left(n_{s}-n_{\bar{s}}\right)}{d \tau} & =0,
\end{aligned}
$$

along with the equilibrium condition,

$$
\mu_{\bar{S}}=-\mu_{S}
$$

Here $e, P$, and $n_{\bar{s}}$ are respectively the energy density, pressure, and $\bar{s}$ density. We connect the hydrodynamic evolution to the initial evolution by using the fact that the baryon and entropy rapidity densities, respectively $d N_{B} / d y$ and $d S / d y$, are approximately invariant after the initial collisions.

In Fig. 2, we show the trajectory followed by the hot matter at $y=0$ in a central $\mathrm{Au}+\mathrm{Au}$ collision at $11.6 \mathrm{GeV} /$ nucleon, using $R_{f}=0.5 \mathrm{fm}$. We use the preliminary results $d N_{B} / d y=120$ and $d N_{\pi^{+}+\pi^{-}} / d y=120$ [16] in the central rapidity region, from which we estimate $d S / d y \sim 1700$ if the freezeout temperature $T_{f}=100-120 \mathrm{MeV}$. At $\tau=l_{N} / 2$ and $\tau=l_{N}, T=162 \mathrm{MeV}$, and the maximum temperature reached is $169 \mathrm{MeV}$, so the hot matter is only superheated by $20-30 \mathrm{MeV}$ during the initial evolution. For comparison, we show also the trajectory obtained with the initial conditions used in Ref. [11], $e \simeq 2 \mathrm{GeV}$ $\mathrm{fm}^{-3}$ and $n_{B} \simeq 0.8 \mathrm{fm}^{-3}$ for $\tau \leq l_{N}$, along with the critical temperature, $T_{c}$. The maximum temperature reached with this second trajectory is $200 \mathrm{MeV}$, significantly higher than for our trajectory. However, our trajectory should reflect the conditions of the thermalized matter, which is most likely to remain at $y=0$, and thus will be accurately determined by rapidity density measurements.

\section{DECAY TO OBSERVED LEPTON AND KAON PAIRS}

There are a number of calculations of the $\phi$ mass and width at finite $T$. The range of predictions for the mass is very wide. Haglin and Gale [6] find that the mass shift $\delta m(T)$ increases slowly and monotonically with increasing $T$, with $\delta m(200 \mathrm{MeV}) \approx 4 \mathrm{MeV}$, while Asakawa and Ko [2, 3] find that $\delta m$ decreases monotonically with $\delta m(190 \mathrm{MeV}) \approx-170$ 
$\mathrm{MeV}$. The range of values for the width, $\Gamma(T)$, is much narrower. Ko and Seibert 《4 and Haglin [7] both find that the RG contribution to $\Gamma(T)$ is approximately $30 \mathrm{MeV}$ at $T=200$ $\mathrm{MeV}$, although it may be as low as $10 \mathrm{MeV}$ if vertex form factors are included [4]. The QGP contribution to the width depends on the dynamics of the two-phase coexistence region, as it is proportional to the fraction of matter in the QGP phase and inversely proportional to the mean QGP droplet radius [5]. In the adiabatic limit (when the nucleation rates for both phases are infinite), the QGP contribution to $\Gamma(T)$ is infinite; however, the contribution to observable quantities is probably small, just a few $\mathrm{MeV}$ in an $\mathrm{Au}+\mathrm{Au}$ collision at $\sqrt{s}=200$ $\mathrm{GeV} /$ nucleon and of order $10 \mathrm{MeV}$ at $\sqrt{s}=10-20 \mathrm{GeV} /$ nucleon.

For the mass, we take two very different parametrizations, following Refs. [6] and [2];

$$
\begin{gathered}
m_{\phi}^{H G}(T)=1020+4(T / 200 \mathrm{MeV})^{4} \mathrm{MeV} \\
m_{\phi}^{A K}(T)=1020-200(T / 200 \mathrm{MeV})^{4} \mathrm{MeV} .
\end{gathered}
$$

We use these masses in the RG phase, and assume that there are no recognizable $\phi$ mesons in the QGP. As the corrections for finite baryon density are uncertain, we neglect them at present. For the width, we use

$$
\Gamma(T)=4.43+25(T / 200 \mathrm{MeV})^{2} \mathrm{MeV} .
$$

The dilepton signal from before freezeout is obtained by convoluting the four-volume per unit rapidity, $\pi r^{2} \tau d \tau$, the $\phi$ density $n_{\phi}(T)$, the thermal phi mass distribution $p(m, T)$, and the decay rate to dileptons, $\Gamma_{l l}$. The post-freezeout contribution is calculated in the same manner, except that we use the vacuum mass and width, and assume that after freezeout the number of $\phi$ mesons decreases exponentially in $\tau$ with the $T=0$ decay rate.

$$
\frac{d N_{l l}}{d m^{2} d y}=\pi r^{2} \Gamma_{l l}\left[\frac{\tau_{f} n_{\phi}\left(T_{f}\right) p(m, 0)}{\Gamma(0)}+\int_{\tau_{0}+l_{N} / 2}^{\tau_{f}} d \tau \tau n_{\phi}(T) p(m, T)\right]
$$

We take $\Gamma_{l l}$ to be constant, evaluate $n_{\phi}$ numerically, and assume a Breit-Wigner mass distribution,

$$
p(m, T)=\frac{m_{\phi}(T) \Gamma(T) / \pi}{\left[m^{2}-m_{\phi}^{2}(T)\right]^{2}+m_{\phi}^{2}(T) \Gamma^{2}(T)} .
$$

We neglect possible dileptons produced before the hot matter is thermalized at proper time $\tau_{0} \approx 1-2 \mathrm{fm} / c$ after the point of maximum overlap; this signal is probably small, and we have no idea what the mass distribution is for these particles. The calculation is simplified by the fact that the lepton mean free path is long, so that virtually all produced pairs escape from the hot matter without interacting.

The kaon signal is calculated in almost the same manner, but with two changes. First, the decay rate to kaons, $\Gamma_{K K}$, depends strongly on the $\phi$ mass. We include this by using

$$
\Gamma_{K K}(T)=\frac{m_{\phi}(0)^{2}\left[m_{\phi}(T)^{2}-4 m_{K}^{2}\right]^{3 / 2}}{m_{\phi}(T)^{2}\left[m_{\phi}(0)^{2}-4 m_{K}^{2}\right]^{3 / 2}} \Gamma(0),
$$


where $m_{K}=495 \mathrm{MeV}$ is the vacuum kaon mass. Second, we fold in the probability for the kaons to escape the hot matter without interaction, which is approximately

$$
\begin{aligned}
\mathcal{P}_{K}(T) & =\frac{2 \pi r_{N} \tau \times \tau_{K}(T) v_{\phi}(T) / 8}{\pi r_{N}^{2} \tau} \\
& =\frac{\tau_{K}(T) v_{\phi}(T)}{4 r_{N}}
\end{aligned}
$$

where $\tau_{K}$ is the mean time between kaon interactions, and $v_{\phi}$ is the mean thermal velocity for $\phi$ mesons. The numerator in the first expression is the product of the surface area of the cylinder and the mean distance from the surface for a pair of emerging kaons (half that for a single kaon), while the denominator is the total volume. As the kaon velocity in the $\phi$ rest frame is not too large, we assume that the kaon and $\phi$ velocities are the same; when the $\phi$ velocity is small enough that this is not true, it is very unlikely that both produced kaons will escape without interaction, so this should produce only a small correction since our escape probability is proportional to $v_{\phi}$ and hence goes to zero in this case.

$$
\frac{d N_{K K}}{d m^{2} d y}=\pi r^{2}\left[\tau_{f} n_{\phi}\left(T_{f}\right) p(m, 0)+\int_{\tau_{0}+l_{N} / 2}^{\tau_{f}} d \tau \tau n_{\phi}(T) \Gamma_{K K}(T) \mathcal{P}_{K K}(T) p(m, T)\right] .
$$

We take $v_{\phi}$ to be the (non-relativistic) rms thermal velocity,

$$
v_{\phi}(T)=\left[\frac{3 T}{m_{\phi}(T)}\right]^{1 / 2}
$$

and use the parametrization

$$
\tau_{K}=3\left(\frac{150 \mathrm{MeV}}{T}\right)^{4} \mathrm{fm} / c
$$

which agrees well with the results of Haglin and Pratt [17].

We show predicted dilepton and dikaon mass spectra in Fig. 3, taking $\tau_{0}=0$ and $T_{f}=100$ $\mathrm{MeV}$. The dikaon spectra differ significantly only far from the $\phi$ peak, when the signal has dropped to less than $1 \%$ of the peak value. As there is large combinatoric background near the peak, the small difference in the dikaon signal will almost certainly be lost, while the difference in the dilepton signal is over $10 \%$ and thus may be detectable.

Note the small secondary peak in the dilepton spectrum at $m \simeq 920 \mathrm{MeV}$ for $m_{\phi}^{A K} 18$. This peak appears because the hot matter spends $1-2 \mathrm{fm} /$ with $T \simeq 160 \mathrm{MeV}$, just as in the case of a first-order phase transition [2, 3] (when the hot matter remains at $T_{c}$ for a long time). However, the peak here reflects the (approximately constant) initial temperature, which results from the spatial extent of the parton wavefunctions, and depends only weakly on $T_{c}$. In the case of a phase transition, the temperature of the hot matter does not remain constant in the coexistence region even though $T_{c}$ is the same for the two phases, because the entropy per baryon is significantly higher in the QGP at fixed $T$ and $\mu_{B}$ [19], leading to smearing of any secondary $\phi$ meson peak created during a phase transition at finite $\mu_{B}$. The larger peak at $m \simeq 1000 \mathrm{MeV}$ is due to the fact that the system is assumed to freeze out instantaneously, so that there is no hot matter with $0<T<T_{f}$ and thus no $\phi$ mesons are seen in the corresponding mass range; this peak will soften and possibly disappear if more realistic dynamics are used. 


\section{CONCLUSIONS}

Although the total signal from dikaons is larger than that from dileptons, the vast majority of the dikaons come from $\phi$ mesons that decay after freezeout of the hot matter. Thus, we see that, not very surprisingly, it is difficult to measure thermal mass shifts by observing strongly interacting particles. Conversely, dileptons make a good probe of thermal mass shifts, since they escape from the hot matter throughout the collision.

There are two reasons for the lack of a strong signal from dikaons. The first is that, once the $\phi$ mass drops appreciably, the decay rate to dikaons vanishes. However, this is not so easily corrected by observing meson decay to lighter particles, such as pions. Although the decay rate then increases, the velocity of the decay products also increases, so that it is greater than the mean thermal velocity of the heavy meson. The decay products then tend to emerge back-to-back in the lab frame, so that the probability of both decay products escaping from the hot matter without interacting is small. It is also possible that the kaon mass drops with increasing $T$ [20], and that the $\phi$ mesons decay to these lighter kaons. However, in this case we expect that the thermal kaons will interact when they leave the hot matter, as they acquire the vacuum kaon mass, and that this interaction will likely remove the pairs from the $\phi$ peak.

As a result of the lack of sensitivity of the dikaon signal to thermal $\phi$ meson properties, it is very important to study dilepton production in ultra-relativistic heavy ion collisions, particularly near vector meson peaks. This is the only likely means for detecting finitetemperature shifts in meson masses and widths. As the study of meson properties may provide valuable insight into the equation of state of hot strongly-interacting matter [21,22, 2], 5 , we would hope to see more experimental attention devoted to these measurements in the future.

\section{ACKNOWLEDGMENTS}

This work was supported in part by the Natural Sciences and Engineering Research Council of Canada, and in part by the FCAR fund of the Québec government. We thank J. Barrette, E. O'Brien, F. Videbaek, and B. Cole for useful comments. 


\section{REFERENCES}

[1] E. Shuryak, Nucl. Phys. A544, 65c (1992); T. Hatsuda, Nucl. Phys. A544, 27c (1992); U. Heinz and K.S. Lee, Nucl. Phys. A544, 503c (1992); C. Schüren et al., Nucl. Phys. A565, 687 (1993); S. Kim and D.K. Sinclair, Phys. Rev. D 48, 4408 (1993); C. Bernard et al., Phys. Rev. D 48, 4419 (1993); C.R. Allton et al., Phys. Rev. D 49, 474 (1994).

[2] M. Asakawa and C.M. Ko, Phys. Lett. B 322, 33 (1994).

[3] M. Asakawa and C.M. Ko, Phys. Rev. C 50, 3064 (1994).

[4] C.M. Ko and D. Seibert, Phys. Rev. C 49, 2198 (1994).

[5] D. Seibert and C.M. Ko, Phys. Rev. C 50, R559 (1994).

[6] K.L. Haglin and C. Gale, Nucl. Phys. B421, 613 (1994).

[7] K. Haglin, Nucl. Phys. A584, 719 (1995).

[8] E859 Collaboration, B. Cole et al., to be published in the Proceedings of Quark Matter '95.

[9] K. Redlich, J. Cleymans, H. Satz, and E. Suhonen, Nucl. Phys. A566, 391c (1994), and references therein.

[10] J. Letessier, A. Tounsi, U. Heinz, J. Sollfrank, and J. Rafelski, Phys. Rev. D, in press.

[11] J.I. Kapusta, A.P. Vischer, and R. Venugopalan, Phys. Rev. D 51, 901 (1995).

[12] D. Seibert and G. Fai, Phys. Rev. C 50, 2532 (1994).

[13] Review of Particle Properties, Particle Data Group, M. Aguilar-Benitez et al., Phys. Rev. D 50, 1173 (1994).

[14] D. Seibert, Phys. Rev. Lett. 67, 12 (1991).

[15] J.D. Bjorken, Phys. Rev. D 27, 140 (1983).

[16] E866 Collaboration, F. Videbaek et al., to be published in the Proceedings of Quark Matter' 95.

[17] K. Haglin and S. Pratt, Phys. Lett. B 328, 255 (1994).

[18] This peak is seen whenever $m_{\phi}\left(T_{0}\right)$ differs significantly from $m_{\phi}(0)$, where $T_{0}$ is the initial temperature (at $\tau=l_{N}$ ).

[19] U. Heinz, K.S. Lee, and M. Rhoades-Brown, Phys. Rev. Lett. 58, 2292 (1987).

[20] D. Lissauer and E. Shuryak, Phys. Lett. B 253, 15 (1991).

[21] P. Siemens and S. Chin, Phys. Rev. Lett. 55, 1266 (1985).

[22] D. Seibert, Phys. Rev. Lett. 68 (1992) 1476; D. Seibert, V.K. Mishra, and G. Fai, Phys. Rev. C 46 (1992) 330. 


\section{FIGURE CAPTIONS}

Fig. 1: $T_{c}$ vs. $\mu_{B}$ using $R_{e x c}=0.5$ and $1 \mathrm{fm}$, for the cases of strangeness equilibrium under the strong interactions and of complete strangeness suppression (denoted "no s").

Fig. 2: Trajectories followed by the hot matter produced in a central $\mathrm{Au}+\mathrm{Au}$ collision at lab energy $11.6 \mathrm{GeV} /$ nucleon. The solid trajectory follows the evolution described in the text, while the KVV trajectory uses the initial conditions from Ref. [11] as the starting point for the hydrodynamic evolution. The matter is assumed to remain in the RG phase, with $R_{e x c}=0.5 \mathrm{fm}$. For comparison, $T_{c}$ is shown for a chemically equilibrated resonance gas with $n_{s}=n_{\bar{s}}$, taking $B=(217 \mathrm{MeV})^{4}$.

Fig. 3: Predicted dikaon (kk) and dilepton (ll) spectra from a central $\mathrm{Au}+\mathrm{Au}$ collision at lab energy $11.6 \mathrm{GeV} /$ nucleon, using $m_{\phi}(T)$ from Refs. [6] and [2], denoted by HG and AK respectively. Evolution of the hot matter proceeds as described in the text, with $R_{e x c}=0.5 \mathrm{fm}$. 
Fig. 1 - Seibert

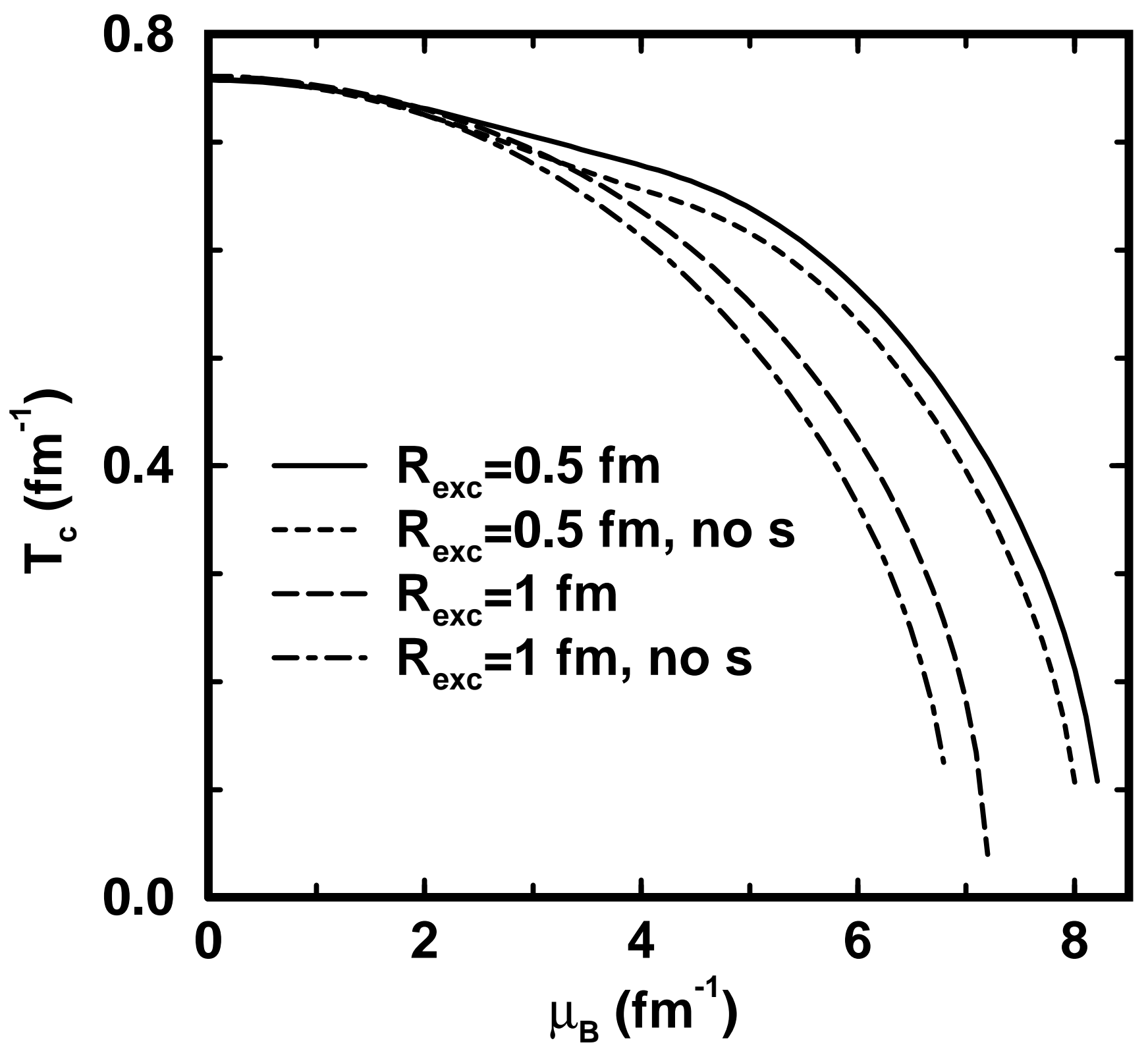


This figure "fig1-1.png" is available in "png" format from: http://arxiv.org/ps/nucl-th/9503014v1 
Fig. 2 - Seibert

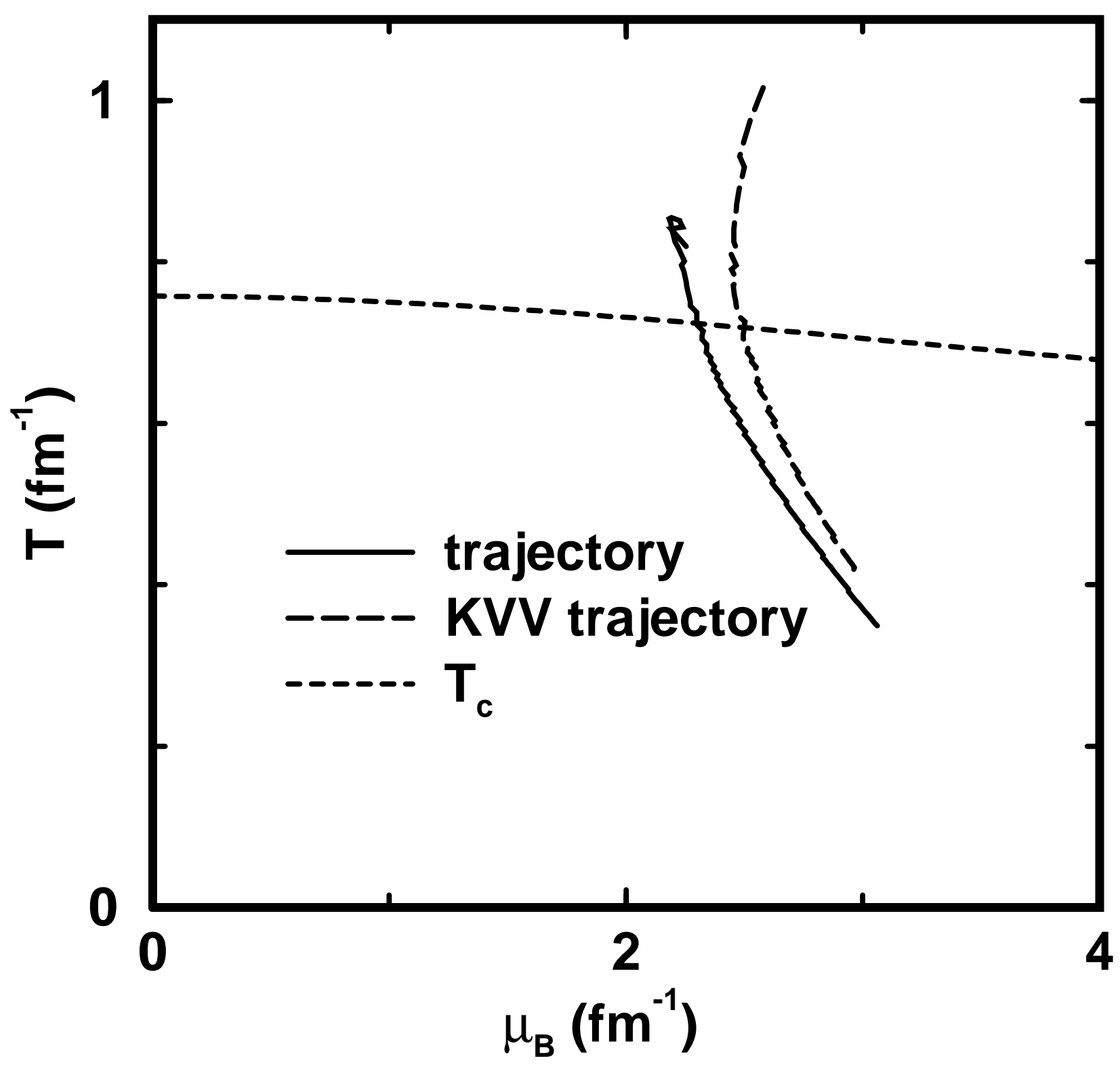


This figure "fig1-2.png" is available in "png" format from: http://arxiv.org/ps/nucl-th/9503014v1 
Fig. 3 - Seibert

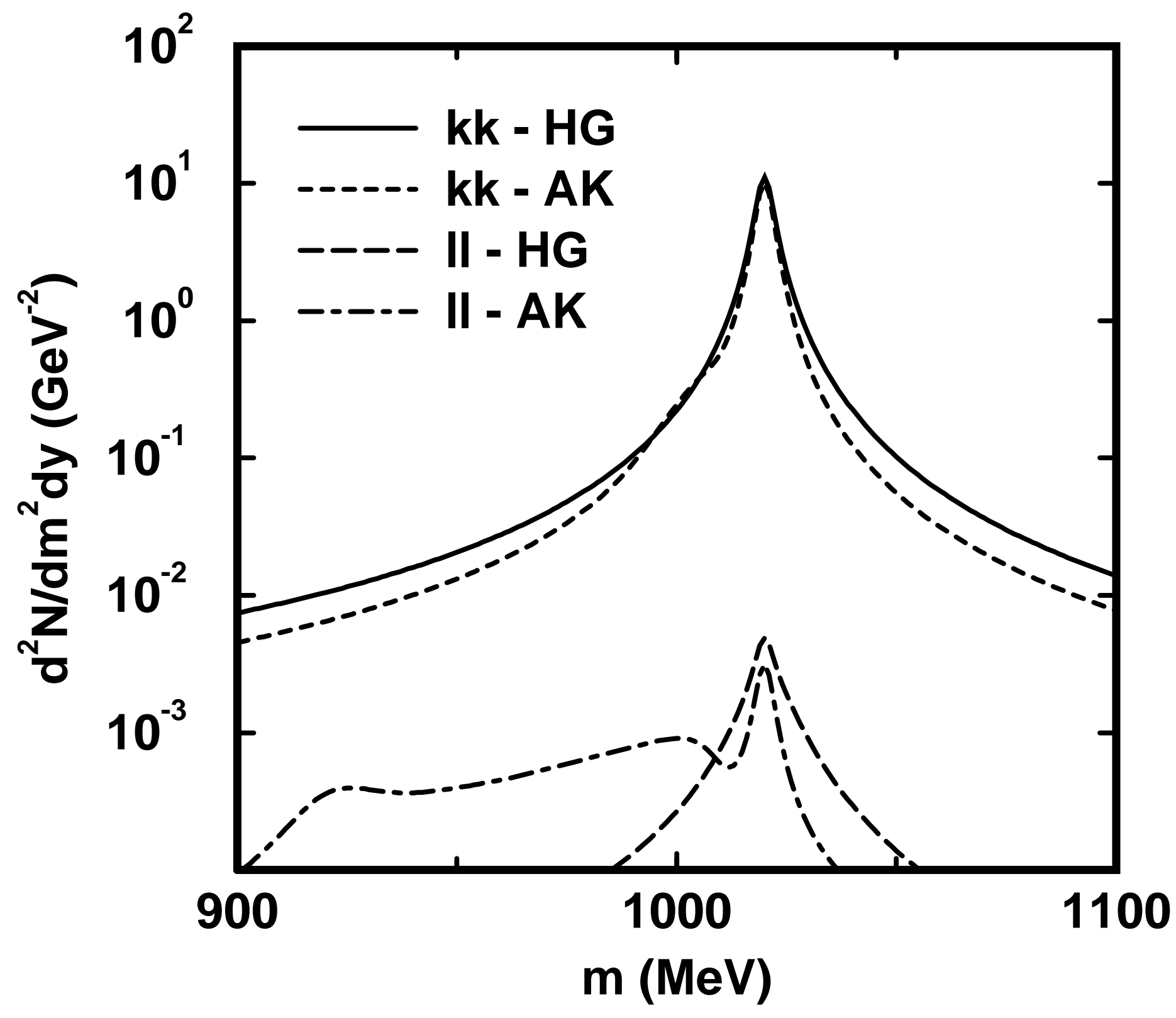


This figure "fig1-3.png" is available in "png" format from: http://arxiv.org/ps/nucl-th/9503014v1 